\title{
TRANSCRIPTION FACTOR AND BONE MARROW STROMAL CELLS IN OSSEOINTEGRATION OF DENTAL IMPLANTS
}

\author{
S.G. Yan ${ }^{1,2,3}$, J. Zhang ${ }^{1,2}$, Q. Tu ${ }^{1}$, J.H. Ye ${ }^{1,4}$, E. Luo ${ }^{1,5}$, M. Schuler ${ }^{6}$, M.M. Dard ${ }^{7}$, Y. Yu ${ }^{8}$, D. Murray ${ }^{1}$, D.L. Cochran ${ }^{9}$, \\ S.H. Kim ${ }^{10}$, P. Yang ${ }^{2, \S}$ and J. Chen ${ }^{1,11, \S, *}$
}

\author{
${ }^{1}$ Division of Oral Biology, Tufts University School of Dental Medicine, Boston, USA \\ ${ }^{2}$ Shandong Provincial Key Lab of Oral Biomedicine, School of Stomatology, Shandong University, Jinan, China \\ ${ }^{3}$ Shandong Academy of Medical Sciences, Jinan, China \\ ${ }^{4}$ Institute of Stomatology, School of Stomatology, Nanjing Medical University, Nanjing China \\ ${ }^{5}$ Department of Oral and Maxillofacial Surgery, School of Stomatology, Sichuan University, Chengdu, China \\ ${ }^{6}$ Institute Straumann AG, Basel, Switzerland \\ ${ }^{7}$ Periodontology and Implant Dentistry, College of Dentistry, New York University, New York City, USA \\ ${ }^{8}$ Department of Dentistry, Zhongshan Hospital Affiliated to Fudan University, Shanghai, China \\ ${ }^{9}$ Department of Periodontics, University of Texas Health Science Centre at San Antonio, San Antonio, USA \\ ${ }^{10}$ Cancer Preventive Material Development Research Centre (CPMDRC) and Institute, College of Oriental Medicine, \\ Kyunghee University, Seoul, South Korea \\ ${ }^{11}$ Department of Anatomy and Cell Biology, Tufts University School of Medicine and \\ Sackler Graduate School of Biomedical Sciences, Boston, USA
}

${ }^{\S}$ These authors contributed equally to the paper

\begin{abstract}
Titanium implants are widely used in dental clinics and orthopaedic surgery. However, bone formation surrounding the implant is relatively slow after inserting the implant. The current study assessed the effects of bone marrow stromal cells (BMSCs) with forced expression of special AT-rich sequence-binding protein 2 (SATB2) on the osseointegration of titanium implants. To determine whether SATB2 overexpression in BMSCs can enhance the osseointegration of implants, BMSCs were infected with the retrovirus encoding Satb2 (pBABE-Satb2) and were locally applied to bone defects before implanting the titanium implants in the mouse femur. Seven and twentyone days after implantation, the femora were isolated for immunohistochemical (IHC) staining, haematoxylin eosin (H\&E) staining, real-time quantitative reverse transcription polymerase chain reaction (qRT-PCR), and micro-computed tomography $(\mu \mathrm{CT})$ analysis. IHC staining analysis revealed that SATB2-overexpressing BMSCs were intensely distributed in the bone tissue surrounding the implant. Histological analysis showed that SATB2overexpressing BMSCs significantly enhanced new bone formation and bone-to-implant contact 3 weeks after implantation. Real-time qRT-PCR results showed that the local delivery of SATB2-overexpressing BMSCs enhanced expression levels of potent osteogenic transcription factors and bone matrix proteins in the implantation sites. $\mu \mathrm{CT}$ analysis demonstrated that SATB2-overexpressing BMSCs significantly increased the density of the newly formed bone surrounding the implant 3 weeks postoperatively. These results conclude that local delivery of SATB2-overexpressing BMSCs significantly accelerates osseointegration of titanium implants. These results provide support for future pharmacological and clinical applications of SATB2, which accelerates bone regeneration around titanium implants.
\end{abstract}

Keywords: Implant; special AT-rich sequence-binding protein 2 (SATB2); osseointegration; bone marrow stromal cells (BMSCs).
*Address for correspondence:

Jake Chen

Division of Oral Biology

Tufts University School of Dental Medicine

One Kneeland Street, Boston MA, 02111, USA

Telephone Number: 617-636-2729

FAX Number: 617-636-0878

E-mail: jk.chen@tufts.edu

\section{Introduction}

Osseointegration, also known as direct bone-toimplant contact (BIC), plays an important role in the long-term success of established titanium implants (Branemark, 1983; Linder et al., 1983). In order to improve osseointegration, various studies on surface properties of the titanium implants and bone regeneration surrounding the implants have been undertaken (Rupp et al., 2006; Sisti et al., 2006; Traini et al., 2008; Faeda et al., 2009). Stem cells, specifically dental pulp stem cells can hook into Biocoral scaffold (Mangano et al., 2011), differentiate into osteoblasts, and form bone on different titanium surface textures (Mangano et al., 2010). Osseointegration of implants is achieved by the activity of osteoblasts (Schneider et al., 2004). Bone marrow stromal cells (BMSCs) contain a subset of stem cells, mesenchymal stem cells that possess multipotential and differentiation features. BMSCs are capable of selfrenewal and can differentiate into several phenotypes including osteoblasts, chondroblasts, and adipocytes (Nussenbaum and Krebsbach, 2006; Robey and Bianco, 2006). Osteoblast progenitors are recruited from bone marrow and are involved in bone regeneration from peripheral circulation (Li et al., 2008). Transplanted BMSCs can be recruited from peripheral circulation to implantation sites and participate in the osseointegration of the titanium implants (Xu et al., 2009). However, there is only a small population of mesenchymal stem cells with osteogenic potential in the implant region, which explains the slow bone regeneration around implants after surgery. 
Special AT-rich sequence-binding protein 2 (SATB2) is a nuclear matrix protein that plays pivotal role in osteoblast differentiation and craniofacial development (Dobreva et al., 2006). SATB2 regulates gene transcription by binding to the nuclear matrix-attachment regions. Satb2 gene knockout mice show multiple craniofacial defects including significant mandible truncation, shortened oral maxillofacial bones, and hyoid bone malformations. It is suggested that in craniofacial reconstruction, SATB2 can be a robust osteoinductive molecule recruiting other transcription factors to form a platform or a molecular node for a transcriptional network. It can synergise, amplify, and thus exponentially augment the activity of multiple osteogenic factors including runt-related transcription factor 2 (Runx2), Osterix (Osx), and activating transcription factor 4 (Atf4) regulating skeletal development and osteoblast differentiation (Dobreva et al., 2006). However, we recently found that SATB2 upregulates Osx expression independent of Runx2, but synergistically enhanced the regulatory effect of Runx2 on Osx promoter (Zhang et al., 2011). Additionally, SATB2 plays an important role in embryonic stem (ES) cell pluripotency (Savarese et al., 2009). It has also been reported that Osterix (Osx) is an upstream regulator of SATB2 and can activate the SATB2 promoter reporter in a dose-dependent manner during bone formation (Tang et al., 2011). Our previous study demonstrated that local delivery of SATB2 enhanced implant osseointegration (Yan et al., 2011).

To determine the prominent properties of Satb2 in bone regeneration, green fluorescent protein (GFP) labelled SATB2-overexpressing BMSCs were locally administrated before implantation. The function of SATB2overexpressing BMSCs in bone formation around the implant was explored.

\section{Materials and Methods}

\section{Plasmids}

The mouse Satb2 cDNA was released from pBs-SK-Satb2 (a gift from Dr. Grosschedl, Gene Centre and Institute of Biochemistry, University of Munich, Munich, Germany), and was ligated into the $B a m H \mathrm{I} / E c o R \mathrm{I}$ sites of a retroviral vector, pBABE-hygro (ID: 1765, Addgene, Cambridge, MA, USA), creating the plasmid pBABE-Satb2 (Yan et al., 2011; Zhang et al., 2011).

\section{Production of high-titre pBABE viral stocks}

The retroviral vectors, pBABE-Satb2 and pBABE-hygro (Zhang et al., 2011), were transfected into HEK-293T (ATCC, Manassas, VA, USA, CRL-11268 ${ }^{\mathrm{TM}}$ ) cells using Lipofectamine transfection reagent (Life Technologies, Grand Island, NY, USA). Forty-eight hours after the transfection, the supernatant was collected, filtered through a $0.45 \mu \mathrm{m}$ filter (Millipore, Bedford, MA, USA), and mixed with $40 \%$ PEG-8000 in phosphate buffered solution (PBS) to reach a final concentration of $12 \%$ (Sigma-Aldrich, St. Louis, MO, USA). The virus-PEG8000 mixtures were incubated on ice for $12 \mathrm{~h}$, and then centrifuged at $4000 \mathrm{rpm}$ $\left(4{ }^{\circ} \mathrm{C}\right)$ for $10 \mathrm{~min}$. The pellets were dissolved in Dulbecco's
Modified Eagle Medium (DMEM) to achieve a viral titre of $10^{8} \mathrm{cfu} / \mathrm{mL}$.

\section{BMSC culture and gene transduction}

BSP-Luc/ACTB-EGFP mice were genetically double labelled with a luciferase reporter gene driven by a bone sialoprotein (BSP) promoter and an enhanced green fluorescent protein (EGFP) driven by a beta-actin promoter (Li et al., 2008). The BMSCs were obtained and cultured as described previously (Wu et al., 2003; Valverde et al., 2005). Briefly, BMSCs from 8-week-old BSP-Luc/ ACTB-EGFP mice were obtained and cultured under non-differentiating conditions (DMEM, with $20 \%$ foetal bovine serum (FBS), $100 \mathrm{mg} / \mathrm{mL}$ penicillin and $100 \mathrm{mg} /$ $\mathrm{mL}$ streptomycin). Afterward, the IVIS 200 imaging system (Xenogen, Alameda, CA, USA) determined GFP expression in the BMSCs.

The BMSCs were infected by the viral supernatant with polybrene at a final concentration of $8 \mu \mathrm{g} / \mathrm{mL}$ for $6 \mathrm{~h}$. BMSCs overexpressing SATB2 were then cultured under non-differentiating conditions (DMEM with $20 \%$ FBS, $100 \mathrm{mg} / \mathrm{mL}$ penicillin and $100 \mathrm{mg} / \mathrm{mL}$ streptomycin). Empty vector infected BMSCs served as a control. Two days after viral infection, $1 \times 10^{6}$ SATB2 overexpressing BMSCs or $1 \times 10^{6}$ empty vector infected BMSCs were resuspended in $100 \mu \mathrm{L}$ of cultured medium and prepared for use. We detected the Osx and Runx2 expression levels to evaluate the BMSC differentiation potential after transduction as previously described (Tu et al., 2006; Zhang et al., 2011).

Real-time quantitative reverse transcription polymerase chain reaction (qRT-PCR) analysis of the infected BMSCs Infected BMSCs (pBABE-Satb2 group and control group) were collected at 2, 9 or 23 days (the time points of the implantation and animals sacrifice) after infection. Total RNA was extracted from the infected BMSCs with TRIzol reagent (Life Technologies), and the first strand cDNA was generated with SuperScript III reverse transcriptase (Life Technologies) and oligo (dT) ${ }_{20}$ primer (Life Technologies). Real-time qRT-PCR analysis was performed using $\mathrm{iQ}^{\mathrm{TM}}$ SYBR Green Supermix (Bio-Rad Laboratories, Hercules, CA, USA) on a Bio-Rad iQ5 thermal cycler (Bio-Rad). In Table 1, the sequences of the primers for amplification of mouse Osx, Runx2, BSP, Osteocalcin (OC), COLI, and glyceraldehyde-3-phosphate dehydrogenase (GAPDH) are listed.

\section{Animal surgery and BMSC application}

The animals were maintained and used in accordance with recommendations in the Guide for the Care and Use of Laboratory Animals, prepared by the Institute on Laboratory Animal Resources, National Research Council (DHHS Publ. NIH 86-23, 1985). The Institutional Animal Use and Care Committee at the Tufts Medical Centre (Boston, MA, USA) approved the animal protocol.

The titanium implants $(1.05 \mathrm{~mm}$ in diameter and $2 \mathrm{~mm}$ in length, Institute Straumann AG, Basel, Switzerland) were SLA (sand-blasting and acid etching) surfaced and the surgery was performed as previously described $(\mathrm{Xu}$ et al., 2009). Briefly, two implantation sites, one in each femur, were prepared on the anterior-distal surface of the 
Table 1. The sequences of the primers for qRT-PCR in the article.

\begin{tabular}{|c|c|}
\hline Primer & Sequence \\
\hline SATB2 & $\begin{array}{l}\text { forward : 5'-GAGATGAGTTGAAGAGGGCTAGTG--3' } \\
\text { reverse : 5'-CCCTGTGTGCGGTTGAAT -3 }\end{array}$ \\
\hline OSX & $\begin{array}{l}\text { forward: 5'-ATGGCGTCCTCTCTGCTTG-3' } \\
\text { reverse: 5'-TGAAAGGTCAGCGTATGGCTT-3' }\end{array}$ \\
\hline RUNX2 & $\begin{array}{l}\text { forward : 5'-CCCAGCCACCTTTACCTACA--3' } \\
\text { reverse : 5'-TATGGAGTGCTGCTGGTCTG -3' }\end{array}$ \\
\hline BSP & $\begin{array}{l}\text { forward: 5'-CAGGGAGGCAGTGACTCTTC-3' } \\
\text { reverse: 5'-AGTGTGGAAAGTGTGGCGTT-3' }\end{array}$ \\
\hline COLI & $\begin{array}{l}\text { forward: 5'-TGACTGGAAGAGCGGAGAGT-3' } \\
\text { reverse: 5'-GTTCGGGCTGATGTACCAGT-3' }\end{array}$ \\
\hline $\mathrm{OCN}$ & $\begin{array}{l}\text { forward : 5'-GCGCTCTGTCTCTCTGACCT-3', } \\
\text { reverse : 5'-GCCGGAGTCTGTTCACTACC-3' }\end{array}$ \\
\hline GAPDH & $\begin{array}{l}\text { forward: 5'-AGGTCGGTGTGAACGGATTTG-3' } \\
\text { reverse: 5'-TGTAGACCATGTAGTTGAGGTCA--3' }\end{array}$ \\
\hline
\end{tabular}

femora in 20 10-week-old B6D2F1 mice (Jax \#100006, Jackson Laboratory, Bar Harbor, ME, USA). The mice were divided randomly into two equal groups. After sequential drilling under cooled sterile saline irrigation with $0.4,0.5$, 0.7 and $1.0 \mathrm{~mm}$ surgical stainless steel twist drills, $3 \mu \mathrm{L}$ of resuspended BMSCs in cultured medium were injected into the drilled holes for implant installation, and the SLAsurfaced implants were press-fitted into the undersized holes. The muscles were carefully sutured over the implant site to cover and stabilise the implants, and the mice were sacrificed 1 and 3 weeks after surgery.

\section{Immunohistochemical (IHC) staining}

After euthanasia, the femora with the implants were isolated, and fixed in $10 \%$ neutral-buffered formalin solution. After decalcification, the implants were gently removed, and the femoral tissues were dehydrated, cleared and embedded in paraffin. Tissue sections, $6 \mu \mathrm{m}$ in thickness, were mounted on glass slides and subjected to IHC and haematoxylin-eosin (H\&E) staining. IHC staining was performed to detect the expression of GFP using the Histostain-SPKit (AEC, Broad Spectrum, Life Technologies). The primary antibody for GFP (Clontech, Palo Alto, CA, USA) was used at a 1:50 dilution following the protocol. The slides were observed under the Nikon Eclipse E600 microscope (Nikon, Tokyo, Japan).

\section{Histomorphometric analysis}

H\&E staining was performed and images were taken under a Nikon Eclipse E600 microscope, and the newly formed bone area, restricted to the $0.5 \mathrm{~mm}$ area surrounding the implant, was measured with Spot Advanced Software (Diagnostic Instruments, Sterling Heights, MI, USA). The percentage of new bone edges in direct contact with the implant surface was also determined (Xu et al., 2009).

\section{Real-time RT-PCR analysis}

The soft tissue and the femoral bone tissues bordering the implant (1 $\mathrm{mm}$ mesial and distal to the implantation site) were carefully dissected, snap-frozen in liquid nitrogen, and the implant was carefully removed. Total RNA was extracted from the bone tissues with TRIzol reagent (Invitrogen, Carlsbad, CA, USA), and Real-time RT-PCR analysis was performed as described previously (Yan et al., 2011).

\section{Micro-computed tomography $(\mu \mathrm{CT})$ analysis}

After euthanasia, the femora with the implants were separated, fixed in $10 \%$ neutral-buffered formalin solution overnight, kept in $70 \%$ ethanol, and scanned with a highresolution $\mu \mathrm{CT}$ (CT40; Scanco Medical, Basserdorf, Switzerland). According to the histomorphometric measurements on the H\&E stained sections, the newly formed bone was restricted to a $0.5 \mathrm{~mm}$ area surrounding the implant. Thus, at a 3D level the Hounsfield Unit (HU) of this newly formed bone area was determined using eFilm Workstation 2.12 (Merge Technologies Inc., Milwaukee, USA) as we reported previously (Xu et al., 2009).

\section{Statistical analysis}

All results are expressed as means \pm standard error of the mean (SEM) of 3 or more independent experiments. One-way ANOVA was used to test significance using the software package Origin 8 (Origin lab, Northampton, MA, USA). Values of $p<0.05$ were considered statistically significant.

\section{Results}

Expression of potent osteogenic transcription factors and bone matrix proteins in infected BMSCs

Real-time qRT-PCR revealed 2 days after infection the mRNA expression levels of SATB2, Osx, and Runx2 were all significantly higher in the pBABE-Satb2 group than in the pBABE-hygro group, however there was no significant difference in the BSP, COLI, and OC mRNA levels between the two groups (Fig. 1a). The expression levels of SATB2, Osx, Runx2, BSP, COLI, and OC were all significantly higher in the pBABE-Satb2 group than in the pBABE-hygro group 9 and 23 days after infection (Fig. 1b,c).

\section{Tracing of exogenous BMSCs}

One week after implantation, IHC staining showed the exogenous BMSCs were randomly distributed and mostly 

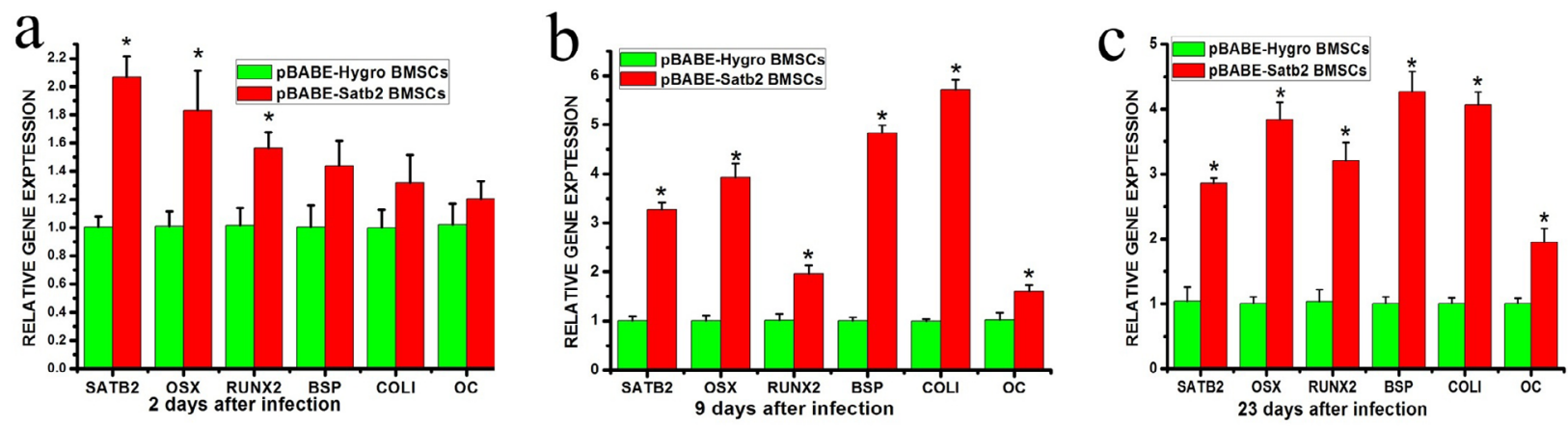

Fig. 1. Bone matrix protein and osteogenic transcription factor expression levels in pBABE-Hygro BMSCs and pBABE-Satb2 BMSCs at (a) 2 days, (b) 9 days, and (c) 23 days after transfection. Data were expressed as mean $\pm \operatorname{SEM}(n=6-8) .{ }^{*} p<0.05$, pBABE-hygro BMSCs $v s$. pBABE-Satb2 BMSCs.
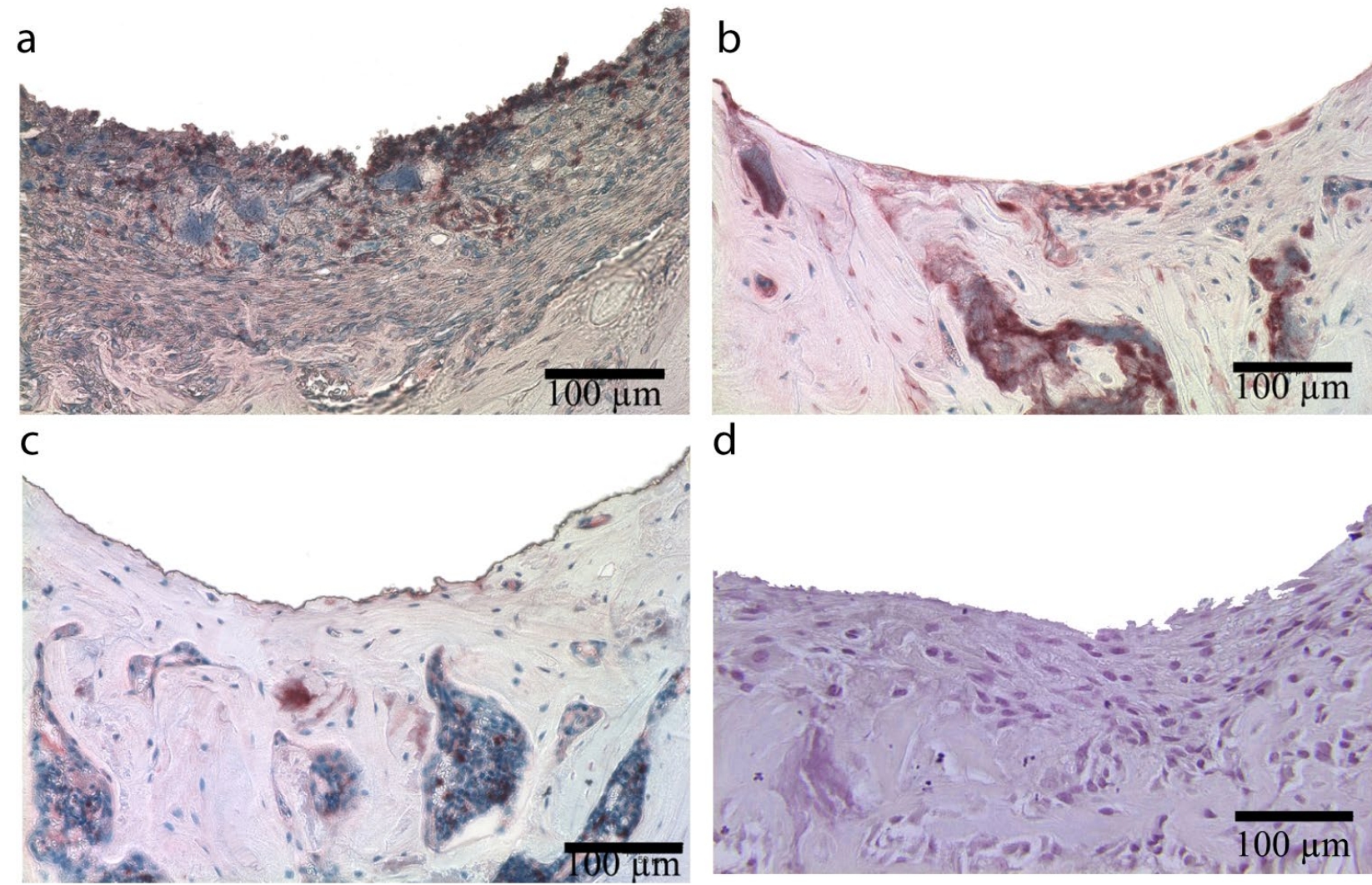

d

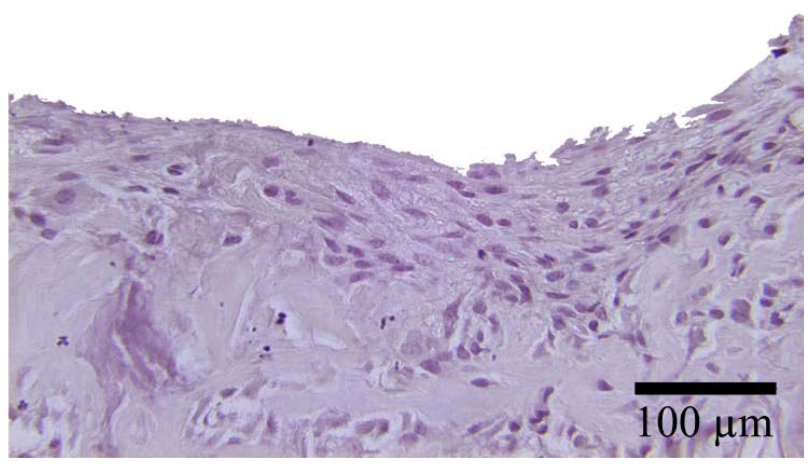

Fig. 2. Immunohistochemical staining for GFP. (a) 1 week after implantation, most of the exogenous BMSCs were found to distribute randomly in the tissue surrounding the implant. (b) 3 weeks after implantation, some of the exogenous BMSCs were embedded in the newly formed bone tissue. (b, c) Positive signals for GFP were found in the bone tissue surrounding the implant. (c) Some exogenous BMSCs could be observed in the bone marrow. (d) Negative control, the negative control did not show any GFP staining.

located in the tissue surrounding and adjacent to the femur implant (Fig. 2a). Three weeks after implantation, positive signals for GFP could be found in the bone tissue surrounding the femur implant (Fig. 2b,c). Specifically, the exogenous BMSCs were found embedded in the newly formed bone tissue (Fig. 2b), and in the bone marrow (Fig. 2c).

\section{Histological analysis of bone regeneration}

In both pBABE-hygro and pBABE-Satb2 BMSCs, newly formed bone was observed 1 week after implantation (Fig. $3 a, b)$. There was no difference detected in the percentage of newly formed bone area and bone-to-implant contact between these two groups (Fig. 3e). Three weeks after implantation, the implants successfully integrated with the host bone and organised lamellar bone formed in both the pBABE-Satb2 and the pBABE-hygro BMSCs (Fig. $3 \mathrm{c}, \mathrm{d})$. Histomorphometric analysis showed that both the percentage of newly formed bone area and bone-to-implant contact increased by about $20 \%$ in the pBABE-Satb2 BMSCs when compared with those in the pBABE-hygro BMSCs (Fig. 3f).

Expression of potent osteogenic transcription factors and bone matrix proteins in bone tissue

One week after implantation, expression levels of SATB2, Osx, Runx2, BSP, and COLI were all significantly higher in the pBABE-Satb2 group than in the pBABE-hygro group 

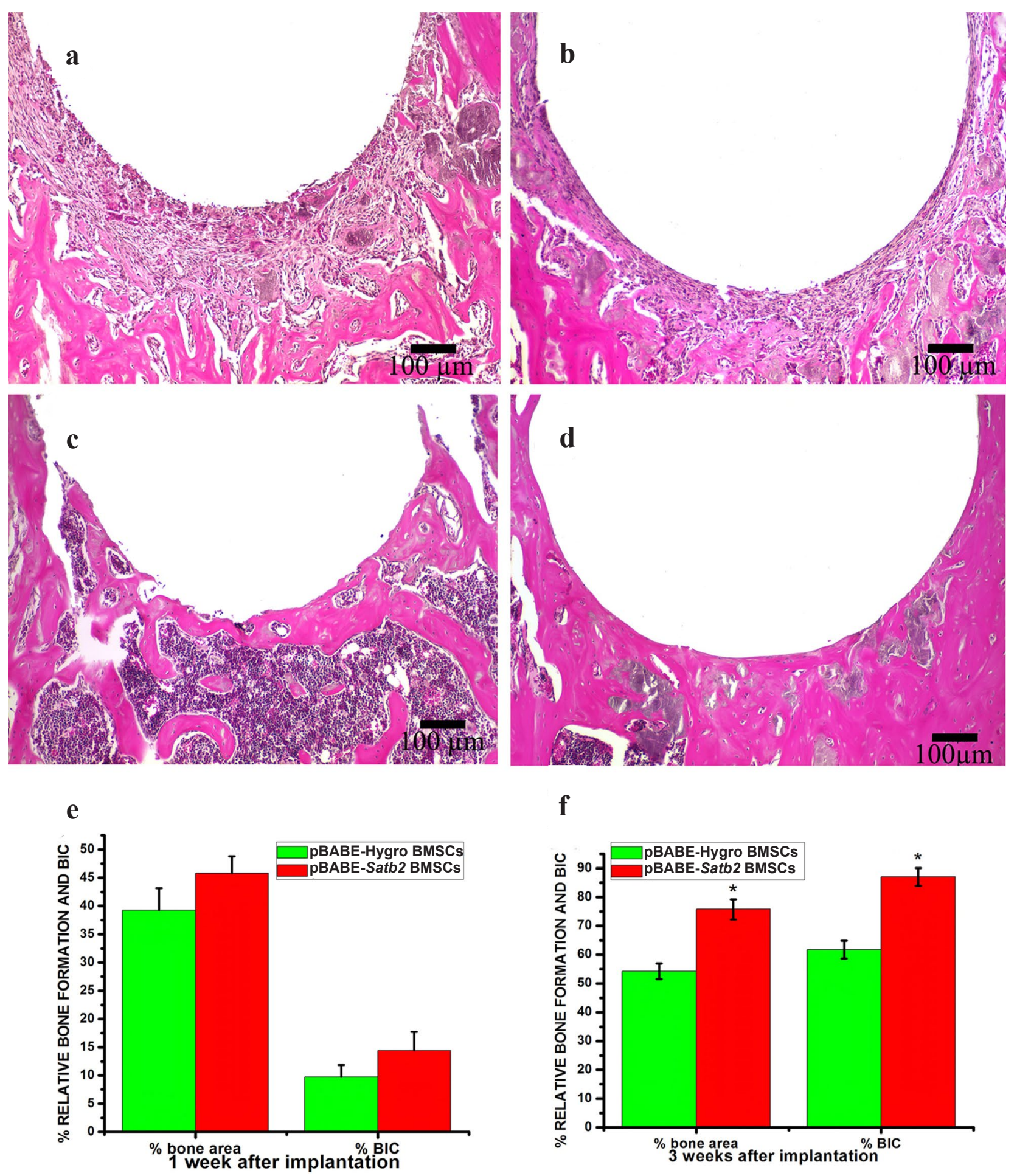

Fig. 3. Histomorphometric analysis. H\&E staining 1 week after implantation showed newly formed bone in (a) pBABE-hygro BMSCs and (b) pBABE-Satb2 BMSCs. No significant difference was detected in the percentage of newly formed bone area and bone-to-implant contact between these two groups (e). H\&E staining 3 weeks after the implantation showed successful integration of the implants with the host bone and organised lamellar bone in (c) pBABE-hygro BMSCs and the (d) pBABE-Satb2 BMSCs. (f) Histomorphometric analysis showed that both the percentage of newly formed bone area and bone-to-implant contact increased by about $20 \%$ in pBABE-Satb2 BMSCs compared with pBABE-hygro BMSCs. Data were expressed as mean \pm SEM $(n=6-8) . * p<0.05$, pBABE-hygro BMSCs vs. pBABE-Satb2 BMSCs. 
a

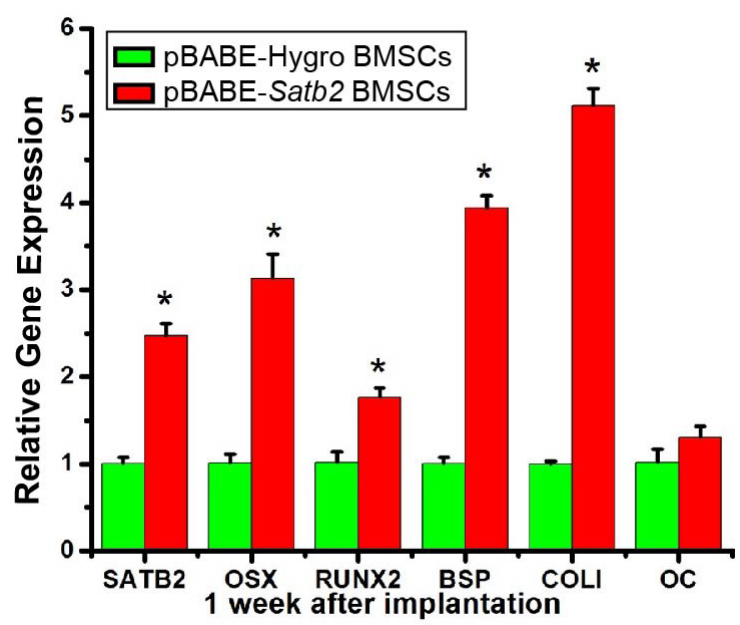

b

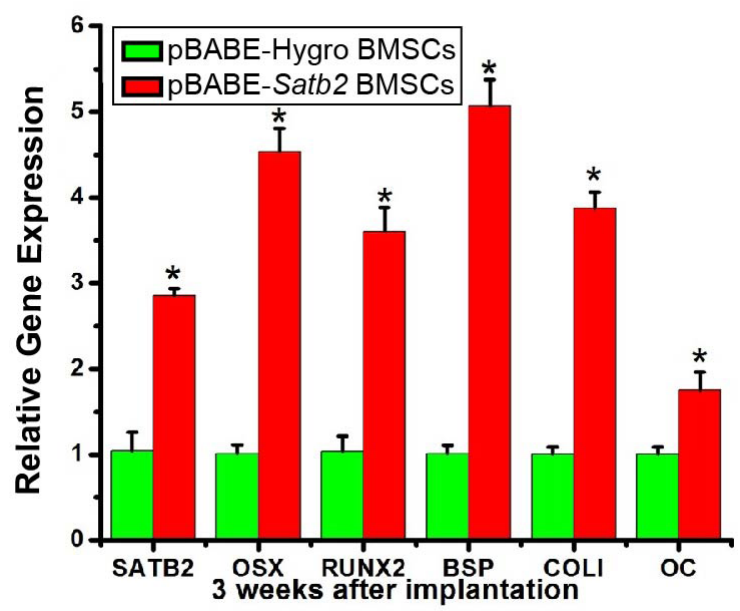

Fig. 4. In newly formed bone tissues, the SATB2 overexpressing BMSCs showed enhanced expression levels of potent osteogenic transcription factors and bone matrix proteins. To evaluate the function of SATB2 overexpressing BMSCs in osteogenic differentiation, qRT-PCR analysis was performed to detect the expression levels of SATB2, Osx, Runx2, BSP, COLI, and OC in the bone tissues surrounding the implant (a) 1 week and (b) 3 weeks after implantation in pBABE-hygro BMSCs and pBABE-Satb2 BMSCs. Data were expressed as mean $\pm \operatorname{SEM}(n=3)$. ${ }^{*} p<0.05$.
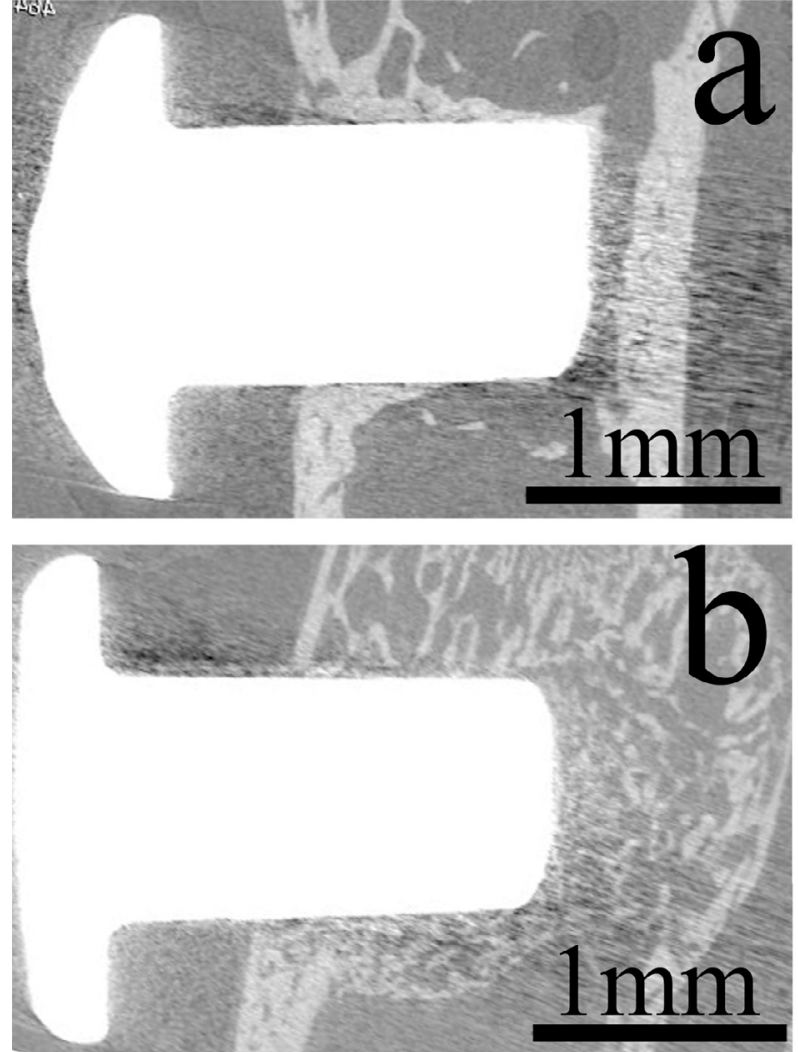

in the newly formed bone tissues, however there was no significant difference in the OC mRNA level between the two groups (Fig. 4a). Three weeks after surgery, the expression levels of SATB2, Osx, Runx2, BSP, COLI, and OC were still significantly higher in the pBABE-Satb2 group than those in the pBABE-hygro group (Fig. 5b).

\section{$\mu \mathrm{CT}$ analysis}

Twenty-one days after implantation, $\mu \mathrm{CT}$ analysis (Fig. 5) showed that the femur implants were surrounded by newly formed bone and successfully anchored with the

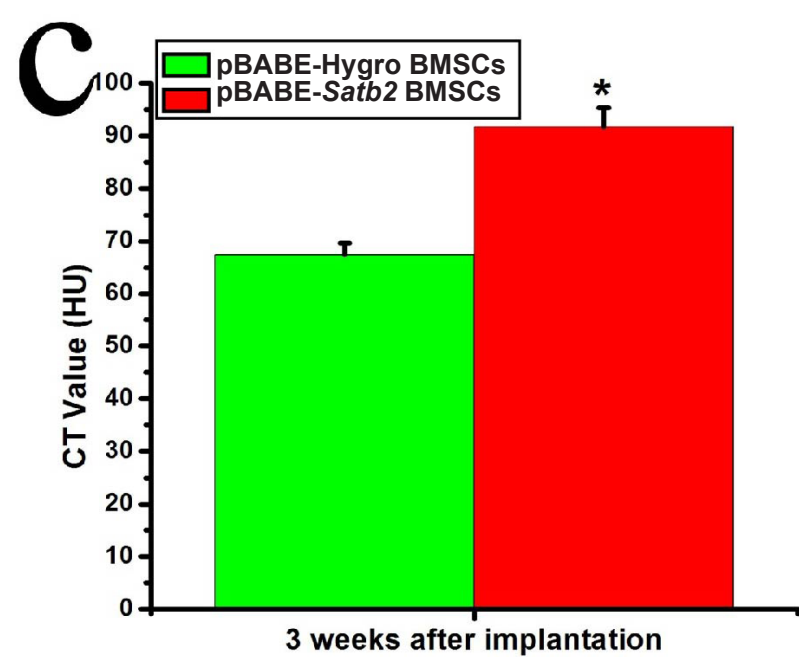

Fig. 5. MicroCT analysis. 21 days after implantation, the implants were successfully anchored in the host bone and surrounded with newly formed bone in (a) pBABE-hygro BMSCs and (b) pBABE-Satb2 BMSCs. (c) The CT value of the newly formed bone surrounding the implant was higher in pBABE-Satb2 BMSCs group than that in pBABE-hygro BMSCs. Data were expressed as mean \pm SEM $(n=3-6)$. $* p<0.05$.

host bone in pBABE-Satb2 BMSCs (Fig. 5a), and pBABEhygro BMSCs (Fig. 5b). SATB2 overexpressing BMSCs markedly enhanced the density of the newly formed bone around the implants (Fig. 5c).

\section{Discussion}

Satb2 plays an important role in craniofacial patterning and bone development, making it a plausible candidate gene for bone tissue engineering techniques. SATB2 
interacts with Runx2 and ATF4, which play essential roles in osteogenic differentiation (Dobreva et al., 2006). This study explored the role of SATB2 overexpressing BMSCs in accelerating implant osseointegration by introducing SATB2 overexpressing BMSCs to the implantation sites and observing the bone-to-implant contact. Though much effort has been made to increase the bone-to-implant contact, bone-to-implant integration is still a challenge. It is generally accepted that the bone forming cells at the titanium implant surface are from the neighbouring host bone. It is also known that the population of mesenchymal stem cells with osteogenic potential is limited to the vicinity of the implant. This can explain why bone regeneration around the implants is relatively slow after placement. The scarceness of bone forming cells in the implant site also poses the difficulty in early implant placement and immediate loading and thus limits the use of titanium implants in certain patients. Our previous study demonstrated that transplanted BMSCs can be recruited from circulation to the implantation sites and participate in implant osseointegration (Xu et al., 2009). Based on this study, BMSCs were labelled with GFP prior to transplantation into the implant sites and GFP staining was performed to determine the BMSC fate.

To investigate the in vitro function of SATB2 in osteogenic differentiation of BMSCs, real-time qRT-PCR analysis was performed. Consistent with previous findings (Zhang et al., 2011), the expression levels of potent osteogenic transcription factors and bone matrix proteins were upregulated by the forced expression of Satb2. This study focused on the function of SATB2 overexpressing BMSCs in early implant osseointegration. It was found that the exogenous SATB2 overexpressing BMSCs were distributed in the tissue surrounding the implant 1 week after the implantation. Most of the exogenous BMSCs were located in the tissue adjacent to the implant. Three weeks after implantation, the exogenous SATB2 overexpressing BMSCs were observed in the newly formed bone tissue. Some of them were embedded in the newly formed bone to form bone cells, and some could be observed in the bone marrow around the implant. Therefore, we conjecture that SATB2 overexpressing BMSCs enhanced bone formation around the implant than compared to the control group.

The expression levels of bone matrix proteins and osteogenic transcription factors were all significantly elevated in bone tissue surrounding the implant 1 and 3 weeks after implantation, which is consistent with in vitro data. Local administration of SATB2-overexpressing BMSCs resulted in increased new bone formation and enhanced implant osseointegration 3 weeks after implantation. These results lay a foundation for future clinical studies to develop novel pharmacological approaches that accelerates osseointegration and bone regeneration surrounding implants. This study explores if exogenous SATB2-overexpressing BMSCs can accelerate the bone formation and implant osseointegration. Future studies could investigate SATB2 as a candidate transcription factor for bone regeneration and early anchor of titanium implantation and study the long-term role of exogenous BMSCs in implant osseointegration.

\section{Conclusion}

SATB2-overexpressing BMSCs significantly enhanced expression levels of osteogenic transcription factors and bone matrix proteins, and accelerated new bone formation and enhanced implant osseointegration. This study provides evidence that BMSCs can contribute to implant osseointegration and SATB2 can be used as a candidate transcription factor for bone tissue engineering and regeneration.

\section{Acknowledgements}

We appreciate the technical support from Nicholas Brady for microCT scanning and data analysis, Jamie Chipman and Terri Naumes for animal care. This work was supported by the combination of the grants: NIH DE16710 and DE21464, International Team of Implantology (ITI), and Institut Straumann AG to J. Chen; National Nature Science Foundation of China (81070835) to P.S Yang; Shandong Provincial Natural Science Foundation (ZR2012HQ028) and China Independent Innovation Foundation of Shandong University (IIFSDU, 2011GN039) to Shiguo Yan.

\section{References}

Branemark PI (1983) Osseointegration and its experimental background. J Prosthet Dent 50: 399-410.

Dobreva G, Chahrour M, Dautzenberg M, Chirivella L, Kanzler B, Farinas I, Karsenty G, Grosschedl R (2006) SATB2 is a multifunctional determinant of craniofacial patterning and osteoblast differentiation. Cell 125: 971986.

Faeda RS, Tavares HS, Sartori R, Guastaldi AC, Marcantonio E, Jr. (2009) Evaluation of titanium implants with surface modification by laser beam. Biomechanical study in rabbit tibias. Braz Oral Res 23: 137-143.

Li S, Tu Q, Zhang J, Stein G, Lian J, Yang PS, Chen J (2008) Systemically transplanted bone marrow stromal cells contributing to bone tissue regeneration. J Cell Physiol 215: 204-209.

Linder L, Albrektsson T, Branemark PI, Hansson HA, Ivarsson B, Jonsson U, Lundstrom I (1983) Electron microscopic analysis of the bone-titanium interface. Acta Orthop Scand 54: 45-52.

Mangano C, De Rosa A, Desiderio V, d'Aquino R, Piattelli A, De Francesco F, Tirino V, Mangano F, Papaccio G (2010) The osteoblastic differentiation of dental pulp stem cells and bone formation on different titanium surface textures. Biomaterials 31: 3543-3551.

Mangano C, Paino F, d'Aquino R, De Rosa A, Iezzi G, Piattelli A, Laino L, Mitsiadis T, Desiderio V, Mangano F, Papaccio G, Tirino V (2011) Human dental pulp stem cells hook into biocoral scaffold forming an engineered biocomplex. PLoS One 6: e18721.

Nussenbaum B, Krebsbach PH (2006) The role of gene therapy for craniofacial and dental tissue engineering. Adv Drug Deliv Rev 58: 577-591. 
Robey PG, Bianco P (2006) The use of adult stem cells in rebuilding the human face. J Am Dent Assoc 137: 961972.

Rupp F, Scheideler L, Olshanska N, de Wild M, Wieland M, Geis-Gerstorfer J (2006) Enhancing surface free energy and hydrophilicity through chemical modification of microstructured titanium implant surfaces. J Biomed Mater Res A 76: 323-334.

Savarese F, Davila A, Nechanitzky R, De La RosaVelazquez I, Pereira CF, Engelke R, Takahashi K, Jenuwein T, Kohwi-Shigematsu T, Fisher AG, Grosschedl R (2009) Satb1 and Satb2 regulate embryonic stem cell differentiation and Nanog expression. Genes Dev 23: 2625-2638.

Schneider GB, Zaharias R, Seabold D, Keller J, Stanford C (2004) Differentiation of preosteoblasts is affected by implant surface microtopographies. J Biomed Mater Res A 69: 462-468.

Sisti KE, Garcia IR, Jr., Guastaldi AC, Antoniolli AC, Rossi R, Brochado Neto Ade L (2006) Analysis of titanium surface irradiated with laser, with and without deposited of durapatite. Acta Cir Bras 21 Suppl 4: 57-62.

Tang W, Li Y, Osimiri L, Zhang C (2011) Osteoblastspecific transcription factor Osterix (Osx) is an upstream regulator of Satb2 during bone formation. J Biol Chem 286: 32995-33002.

Traini T, Mangano C, Sammons RL, Mangano F, Macchi A, Piattelli A. (2008) Direct laser metal sintering as a new approach to fabrication of an isoelastic functionally graded material for manufacture of porous titanium dental implants. Dent Mater 24: 1525-1533.

Tu Q, Valverde P, Chen J (2006) Osterix enhances proliferation and osteogenic potential of bone marrow stromal cells. Biochem Biophys Res Commun 341: 1257 1265.

Valverde P, Tu Q, Chen J (2005) BSP and RANKL induce osteoclastogenesis and bone resorption synergistically. J Bone Miner Res 20: 1669-1679.

Wu X, McKenna MA, Feng X, Nagy TR, McDonald JM (2003) Osteoclast apoptosis: the role of Fas in vivo and in vitro. Endocrinology 144: 5545-5555.

Xu B, Zhang J, Brewer E, Tu Q, Yu L, Tang J, Krebsbach P, Wieland M, Chen J (2009) Osterix enhances BMSC-associated osseointegration of implants. J Dent Res 88: 1003-1007.

Yan SG, Zhang J, Tu QS, Ye JH, Luo E, Schuler M, Kim MS, Griffin T, Zhao J, Duan XJ, Cochran DJ, Murray D, Yang PS, Chen J (2011) Enhanced osseointegration of titanium implant through the local delivery of transcription factor SATB2. Biomaterials 32: 8676-8683.

Zhang J, Tu Q, Grosschedl R, Kim MS, Griffin T, Drissi H, Yang P, Chen J (2011) Roles of SATB2 in osteogenic differentiation and bone regeneration. Tissue Eng Part A 17: 1767-1776.

\section{Discussion with Reviewers}

L. Jimenez-Rojo: Please specify the novelties contained in this article with respect to your previous study (Yan et al., 2011, text reference).
Authors: In our previous study, the RCAS viral system was found to function in a relatively shorter time period. The RCAS-Satb2 group showed dramatic increases in mRNA levels of SATB2, Osx, Runx2, BSP, COLI, and $\mathrm{OC}$ at 1 week after implantation when compared with the RCAS group. In contrast, only moderate changes in the mRNA levels of these genes were observed between the pBABE-Satb2 group and the pBABE-hygro group. Three weeks after surgery, the expression levels of SATB2, Osx, Runx2, BSP, COLI, and OC were still significantly higher in the pBABE-Satb2 group than those in the pBABE-hygro group, while there was no significant difference between RCAS-Satb2 and RCAS group. pBABE-Satb2 has a longer effect on the targeted gene expression compared with RCAS-Satb2. When RCAS-Satb2 was applied, only BSP-expressing cells, which had already committed to the osteoblastic lineage were infected and overexpressed SATB2. In contrast, pBABE-Satb2 could infect all local cells including adult stem cells with the ability of selfrenewal and multi-potential of differentiation, which may prolong and enlarge the effect of the original SATB2 infection until a later time point. Based on our previous study, we hypothesised that exogenous bone forming cells with forced expression of SATB2 might elevate bone forming around the implant dramatically. So, this study was carried out and the functions of BMSCs with forced expression of SATB2 were detected in vitro and in vivo, and it was found that BMSCs can contribute to osseointegration of dental implants and SATB2 can be used as a candidate transcription factor for bone tissue engineering and regeneration.

G. Papaccio: The aim of this work is to describe a new system for improving osseointegration of dental implants. However, authors implant them in the femur instead of doing it in the alveolar bone. This may not be a good strategy, since these bones have different characteristics and the attachment of the tooth depends on more elements (periodontal ligament) that cannot be assessed if the implantation is done in the femur.

Authors: Most of the authors are from the dental school and focus on the early anchor of dental implants. In this study, we focused on the function of SATB2-overexpressing BMSCs in titanium implant osseointegration. This femur model characterised an in vivo dental implant experimental model to study implant osseointegration, as others have reported (Rahal et al., 1993; Nociti et al., 1997; Mushahary et al., 2013, additional references). Additionally, the periodontal ligament is not involved in the process of implant osseointegration.

G. Papaccio: BMSCs significantly enhanced expression levels of osteogenic transcription factors and bone matrix proteins around the implantation site? The authors did not demonstrate if the BMSCs are inducing the expression of these factors in the endogenous cells. The increase in these factors may be true only for the Satb2-transfected cells that actually may be already differentiated osteoblasts at the moment of the implantation. Please clarify this issue! Authors: SATB2 is a nuclear matrix protein and regulates gene transcription by binding to the nuclear matrix- 
attachment regions. In this study, BMSCs transfected with pBABE-hygro virus served as the control. The difference between the two groups is whether the BMSCs were forced to express SATB2. We did not detect if the endogenous cells around the implant site was involved in inducing the expression of the factors mentioned in the current experiment.

G. Papaccio: Are the BMSCs already differentiated to osteoblasts at the moment of the implantations, Should you not have included TRAP staining?

Authors: This should certainly have been done. However, we focused this experiment on bone forming and osseointegration around the implants, therefore TRAP staining was not included.

\section{Additional References}

Mushahary D, Sravanthi R, Li Y, Kumar MJ, Harishankar N, Hodgson PD, Wen C, Pande G (2013) Zirconium, calcium, and strontium contents in magnesium based biodegradable alloys modulate the efficiency of implant-induced osseointegration. Int J Nanomed 8: 2887 2902.

Nociti Jr FH, Sallum AW, Sallum EA, Bozzo L (1997) Titanium implants in rabbit femur: a histologic evaluation. Braz Dent J 8: 105-111.

Rahal MD, Branemark PI, Osmond DG (1993) Response of bone marrow to titanium implants: osseointegration and the establishment of a bone marrow-titanium interface in mice. Int J Oral Maxillofac Implants 8: 573-579. 